\title{
Comparison of Risk Factors for Bilateral and Unilateral Eye Involvement in Normal-Tension Glaucoma
}

\author{
Cinoo Kim and Tae-Woo Kim
}

Purpose. To compare the risk factors for bilateral and unilateral disease in normal-tension glaucoma (NTG).

Methods. The study analyzed data from consecutive NTG patients with either bilateral $(n=194)$ or unilateral $(n=193)$ disease. Multivariate regression models were used to evaluate the following potential risk factors for bilateral eye involvement: age, sex, central corneal thickness, mean deviation in standard automated perimetry, mean and fluctuation of diurnal intraocular pressure (IOP) without glaucoma medication, spherical equivalent, family history of glaucoma, and underlying systemic disease such as diabetes mellitus, hypertension, hypercholesterolemia, ischemic heart disease, previous cerebrovascular accident, migraine, and cold extremities.

Results. Multivariate analysis showed that worse mean deviation in the first affected eye (odds ratio [OR], 1.19/1 dB decrease; $P<0.001$ ), diabetes mellitus (OR, 2.31; $P=0.004)$, previous cerebrovascular accident (OR, 4.27; $P=0.039)$, and mean IOP greater than $14 \mathrm{~mm} \mathrm{Hg}$ in the less affected eye (OR, $1.66 ; P=0.030$ ) were significantly associated with bilateral eye involvement in NTG.

Conclusions. Besides being an indicator of disease severity (worse mean deviation), diabetes mellitus, previous cerebrovascular accident, and IOP were associated with bilateral eye involvement. These data suggest that systemic vascular factors and IOP play significant roles in the pathogenesis of NTG. It appears that although systemic vascular factors increase the susceptibility of the optic nerve, a certain level of pressureinduced stress is also required to cause glaucomatous optic neuropathy. (Invest Ophthalmol Vis Sci. 2009;50:1215-1220) DOI:10.1167/iovs.08-1886

Sudies have shown that glaucoma is associated with many $\checkmark$ risk factors, including age, ${ }^{1,2}$ race,${ }^{2,3} \mathrm{sex}^{2}$ positive family history, ${ }^{4}$ myopia,${ }^{5,6}$ diabetes mellitus, ${ }^{7-9}$ hypertension, ${ }^{10}$ disc hemorrhage, ${ }^{11,12}$ and large diurnal fluctuations in intraocular pressure (IOP). ${ }^{13}$ Recently, central corneal thickness (CCT) has been recognized in the Ocular Hypertension Treatment Study as a significant risk factor for the progression of ocular hypertension to primary open-angle glaucoma (POAG). ${ }^{14}$

Normal-tension glaucoma (NTG) is considered to be more strongly affected by non-IOP factors, particularly vascular factors, than high-tension glaucoma. Factors such as migraine, Raynaud phenomenon, nocturnal hypotension, and history of hypovolemic shock have been shown to be associated with

From the Department of Ophthalmology, Seoul National University Bundang Hospital, Seoul National University College of Medicine, Seoul, Korea.

Submitted for publication February 15, 2008; revised July 27 and September 19, 2008; accepted January 7, 2009.

Disclosure: C. Kim, None; T.-W. Kim, None

The publication costs of this article were defrayed in part by page charge payment. This article must therefore be marked "advertisement" in accordance with 18 U.S.C. $\$ 1734$ solely to indicate this fact.

Corresponding author: Tae-Woo Kim, Department of Ophthalmology, Seoul National University Bundang Hospital, Seoul National University College of Medicine, 300 Gumi-dong, Bundang-gu, Seongnam, Gyeonggi-do 463-707, Korea; twkim7@snu.ac.kr.
NTG. ${ }^{15-17}$ Associations between NTG and sleep apnea ${ }^{18,19}$ or reduced cerebrovascular blood flow ${ }^{20}$ have also been reported.

An intriguing finding regarding the pathophysiology of NTG is the frequent observation that some patients have advanced unilateral NTG without the involvement of the other eye, whereas other patients show involvement of both eyes from the early stage of disease. This finding suggests that there might be two different categories of risk factors: those related to an individual eye (eye-related factors) and systemic factors that influence both eyes simultaneously (patient-related factors). It may be reasonably hypothesized that when eye-related factors play a major role, NTG develops unilaterally in the affected eye while the other eye remains unaffected. On the other hand, when patient-related factors play a major role, NTG may show bilateral involvement from the early stage of disease. Identifying and classifying these factors could contribute to a further understanding of the pathophysiology of NTG. The aim of this study was to determine the risk factors for bilateral involvement in NTG.

\section{Materials ANd Methods}

\section{Patients}

The study involved consecutive patients with newly diagnosed NTG who presented at the glaucoma clinic of the Department of Ophthalmology, Seoul National University Bundang Hospital (Seoul, Korea), between 2003 and 2006. The study was approved by the Seoul National University Bundang Hospital institutional review board (IRB no. B-0805-057-007) and conformed to the Declaration of Helsinki. Informed consent was obtained from all subjects.

Before the study, all patients underwent standard face-to-face interviews to collect medical histories and complete ophthalmic examinations, including visual acuity measurement, refraction test, slit lamp biomicroscopy, gonioscopy, Goldmann applanation tonometry, dilated stereoscopic examination of the optic disc, red-free fundus photography, CCT measurement by corneal topography (Orbscan, version 3.12; Bausch \& Lomb, Rochester, NY), retinal nerve fiber layer thickness measurement by optical coherence tomography (Stratus OCT instrument; Carl-Zeiss Meditec, Dublin, CA), and visual field examination by standard automated perimetry.

Five baseline IOP readings were taken for each patient on the same day, between 9:00 am and 5:00 pm. Stereoscopic photographs were taken with a digital camera (EOS D60; Canon, Utsunomiyashi, Tochigiken, Japan) during the same sessions when red-free fundus photography was performed. Photographs were assessed on an LCD monitor with a stereoscopic viewer (Screen-Vu Stereo Viewer; Berezin Stereo Photography Products, Mission Viejo, CA). Vertical cup-to-disc ratios were measured with stereoscopic optic disc photographs and computer-aided planimetry. The disc margin was defined as the inner margin of the scleral ring. The cup margin was drawn at the inflection point in the slope of the neural rim. Red-free fundus photographs were acquired with a digital camera after maximal pupil dilation. Sixtydegree wide-angle views of the optic disc, carefully focused on the retina with the built-in split-lines focusing device and centered between the fovea and the optic disc, were obtained and reviewed on an LCD monitor.

Visual field analysis was performed with the Swedish Interactive Threshold Algorithm standard of the Humphrey Field Analyzer II 750 
(Carl-Zeiss Meditec) and the Humphrey 24-2 test pattern. Glaucomatous visual field loss was defined as two or more adjacent points of $\geq 5$ decibel (dB) loss or one or more points of $\geq 10 \mathrm{~dB}$ loss from the age-corrected normal reference values, ${ }^{21}$ a pattern SD outside the $95 \%$ normal limits, or a glaucoma hemifield test result outside normal limits. For all criteria, confirmation on a second visual field was required. Only reliable visual fields (fixation losses, false positives, and false negatives all $\leq 20 \%$ ) were included.

All patients underwent diurnal curve measurement to rule out unexpected IOP spikes and to determine mean and magnitude of fluctuation of IOP values throughout the day. To be eligible for the study, patients had to have unilateral or bilateral NTG defined according to the following criteria: IOP consistently $<21 \mathrm{~mm} \mathrm{Hg}$ in an eye without antiglaucoma medication; characteristic glaucomatous optic nerve head damage, such as notch and neuroretinal rim thinning; glaucomatous visual field loss; no neurologic disorder that could affect the optic nerve; and open iridocorneal angle and no abnormal chamber angle structure on gonioscopic examination.

Patients were excluded if they had visual acuity worse than 20/40, any diurnal IOP curve measurement $\geq 21 \mathrm{~mm} \mathrm{Hg}$ in either eye, any other ophthalmic disease including media opacity or retinal disease that could result in visual field defects, history of intraocular surgery or laser treatment except for cataract surgery, or history of refractive ablation surgery that could affect corneal thickness.

History of cerebrovascular accident was not an exclusion criterion, but visual field loss attributed to the cerebrovascular accident (i.e., homonymous loss respecting the vertical meridian) was.

\section{Study Variables}

The following factors were included as potential risk factors for bilateral eye involvement: age, sex, spherical equivalent (SE), CCT, mean and fluctuation of diurnal IOP measurement without glaucoma medication, mean deviation of visual field test of the first affected eye (affected eye in the unilateral NTG group; in the bilateral NTG group, the eye with worse mean deviation was considered to represent the first affected eye), family history of glaucoma, and underlying systemic disease (e.g., systemic hypertension, diabetes mellitus, hypercholesterolemia, ischemic heart disease, previous cerebrovascular accident including ischemic or hemorrhagic stroke and transient ischemic attack, migraine, and cold extremities). Information on systemic diseases was obtained from medical records and was confirmed by the class of medication used. Among risk factors, medical histories were included in the analysis only if their presence predated the NTG diagnosis date. If the existing information was considered insufficient, patients were further interviewed at clinic visits during the study period or by telephone. The magnitude of diurnal IOP fluctuation was calculated as the difference between the maximum and minimum diurnal IOP measurements in the absence of glaucoma medication. Interocular differences in mean and fluctuation of diurnal IOP, CCT, and SE were calculated by subtracting values of the first affected eye from that of the less affected eye (the unaffected eye in the unilateral NTG group and the eye with better mean deviation in the bilateral NTG group).

\section{Statistical Analysis}

Statistical analysis of the data was performed using SPSS version 15.0 (SPSS Inc., Chicago, IL). The $\chi^{2}$ test was used to compare possible associations between two categorical variables. For continuous variables, Student's $t$-test and ANOVA were used. Variables with statistical significance of less than 0.10 on univariate analysis were included in multivariate modeling. The enter method of multiple logistic regression was applied for multivariate analysis; $P<0.05$ was considered to indicate a significant difference.

\section{Results}

This study involved 483 patients diagnosed with NTG. Of those, 35 had other ocular disease (age-related macular degen- eration [ $n=17]$, diabetic retinopathy $[n=10]$, branch retinal vein occlusion [ $n=5]$, uveitis $[n=3]$ ) and were excluded from further analysis. Also excluded were 12 patients with visual acuity worse than $20 / 40$, eight patients who had undergone refractive corneal ablation surgery, and three who had undergone intraocular surgery. In addition, 38 subjects who had been diagnosed elsewhere and had undergone some antiglaucoma treatment were excluded. Therefore, the final analysis involved 387 patients.

Of the 387 study patients, 193 had unilateral NTG and 194 had bilateral NTG. All patients were Korean. In the unilateral NTG group, the left eye showed a tendency to be more commonly affected ( $57 \%$ vs. $43 \% ; P=0.084$; binomial test). Similarly, in the bilateral NTG group, more advanced visual field defects tended to be more commonly observed in the left eye ( $56 \%$ vs. $44 \% ; P=0.098$; binomial test).

Of the 193 patients with unilateral NTG, 31 (16.1\%) had a mean deviation worse than $-6 \mathrm{~dB}$ in the affected eye. In contrast, $58(29.9 \%)$ of the 194 bilateral NTG patients had a mean deviation better than $-3 \mathrm{~dB}$ in both eyes.

Information regarding medical history was obtained primarily through preexisting records or from interviews undertaken during the study period. In 10 patients, missing information was obtained by telephone interviews after verbal consent was obtained.

Table 1 compares data obtained from the unilateral and bilateral NTG groups and shows the results of univariate analyses for association with bilateral NTG of all predictor valuables. Mean deviation in the first affected eye $(-3.2 \pm 3.5 \mathrm{~dB}$ vs. $-7.1 \pm 6.4 \mathrm{~dB} ; P<0.001)$, mean IOP of the less affected eye $(14.1 \pm 2.7 \mathrm{~mm} \mathrm{Hg}$ vs. $14.9 \pm 2.9 \mathrm{~mm} \mathrm{Hg} ; P=0.010)$, IOP greater than $14 \mathrm{~mm} \mathrm{Hg}$ in the less affected eye $(39.9 \%$ vs. $54.1 \% ; P=0.005)$, diabetes mellitus ( $14.5 \%$ vs. $28.4 \% ; P=$ $0.001)$, and previous cerebrovascular accident (1.6\% vs. $7.2 \%$; $P=0.007)$ were significantly related to bilateral eye involvement by univariate analysis.

Table 2 shows the comparison of ocular factors between the first affected and less affected eyes in the unilateral and bilateral NTG groups. There were no significant differences in the mean and fluctuation of IOP, CCT, and average SE between eyes in each group (Table 2).

Multivariate analysis also showed that worse mean deviation of the first affected eye (odds ratio [OR], 1.19/1 dB decrease; $P<0.001$ ), diabetes mellitus (OR, 2.31; $P=0.004$ ), previous cerebrovascular accident (OR, 4.27; $P=0.039)$, and mean IOP greater than $14 \mathrm{~mm} \mathrm{Hg}$ in the less affected eye (OR, 1.66; $P=$ $0.030)$ were significantly associated with bilateral eye involvement in NTG (Table 3).

\section{Discussion}

This study demonstrates that NTG patients show a greater tendency to bilateral eye involvement when they have worse mean deviation in the first affected eye, diabetes mellitus, previous cerebrovascular accident, or IOP $>14 \mathrm{~mm} \mathrm{Hg}$ in the less affected eye.

In general, primary glaucoma is believed to be a bilateral disease. Although the condition may present unilaterally, it is expected that the other eye will eventually be affected as the disease advances. It is thus not surprising that worse mean deviation in the first affected eye, an indicator of disease severity, is significantly associated with bilateral eye involvement.

In the present study, diabetes mellitus was shown to be associated with bilateral involvement, suggesting that diabetes mellitus may influence eyes to become more susceptible to glaucomatous damage. Although the association of diabetes 
TABLE 1. Comparisons between Unilateral and Bilateral NTG Groups and Univariate Analysis of Candidate Factors for Bilateral NTG

\begin{tabular}{|c|c|c|c|c|c|}
\hline Possible Predictor Variables & $\begin{array}{c}\text { Unilateral NTG } \\
\text { Group }(n=193)\end{array}$ & $\begin{array}{c}\text { Bilateral NTG } \\
\text { Group }(n=194)\end{array}$ & $\begin{array}{l}\text { Odds } \\
\text { Ratio }\end{array}$ & $\begin{array}{l}\text { 95\% Confidence } \\
\text { Interval }\end{array}$ & $\boldsymbol{P}^{*}$ \\
\hline Age (y) & $60.7 \pm 10.9$ & $61.9 \pm 12.2$ & 1.01 & $0.99-1.03$ & 0.291 \\
\hline Sex (M:F) & $117: 76$ & $109: 85$ & 0.83 & $0.56-1.25$ & 0.376 \\
\hline \multicolumn{6}{|l|}{ CCT $(\mu \mathrm{m})$} \\
\hline Mean & $564.6 \pm 40.1$ & $563.9 \pm 38.0$ & 0.99 & $0.99-1.01$ & 0.816 \\
\hline Interocular difference & $5.69 \pm 20.78$ & $3.34 \pm 11.50$ & 0.98 & $0.97-1.02$ & 0.472 \\
\hline $\mathrm{CCT}<530, n(\%)$ & $30(15.5)$ & $32(16.5)$ & 1.04 & $0.60-1.81$ & 0.878 \\
\hline Mean deviation (dB) & $-3.2 \pm 3.5$ & $-7.1 \pm 6.4$ & 1.19 & $1.13-1.25$ & $<0.001 \ddagger$ \\
\hline \multicolumn{6}{|l|}{ IOP diurnal variation $(\mathrm{mm} \mathrm{Hg}) \dagger$} \\
\hline Mean & $14.1 \pm 2.7$ & $14.9 \pm 2.9$ & 1.10 & $1.02-1.19$ & $0.010 \ddagger$ \\
\hline Interocular difference & $-0.37 \pm 1.56$ & $-0.06 \pm 1.34$ & 1.16 & $1.01-1.34$ & $0.037 \ddagger$ \\
\hline Mean IOP $>14 \mathrm{~mm} \mathrm{Hg}, n(\%)$ & $77(39.9)$ & $105(54.1)$ & 1.78 & $1.19-2.66$ & $0.005 \ddagger$ \\
\hline \multicolumn{6}{|l|}{ IOP fluctuation (mm $\mathrm{Hg})$} \\
\hline Mean & $2.39 \pm 1.51$ & $2.44 \pm 1.11$ & 1.02 & $0.84-1.24$ & 0.811 \\
\hline Interocular difference & $-0.07 \pm 1.14$ & $-0.08 \pm 0.92$ & 0.98 & $0.80-1.19$ & 0.819 \\
\hline Fluctuation $>3, n(\%)$ & $32(16.6)$ & $14(7.2)$ & 0.53 & $0.27-1.06$ & 0.071 \\
\hline \multicolumn{6}{|l|}{ SE (diopter) } \\
\hline Mean & $-0.51 \pm 1.05$ & $-1.13 \pm 1.75$ & 0.89 & $0.71-1.13$ & 0.350 \\
\hline Interocular difference & $-0.07 \pm 0.47$ & $0.10 \pm 1.65$ & 1.09 & $0.69-1.71$ & 0.730 \\
\hline $\mathrm{SE}<-6.0, n(\%)$ & $14(7.6)$ & $15(7.7)$ & 1.04 & $0.55-2.09$ & 0.831 \\
\hline Family history of glaucoma, $n$ (\%) & $6(3.1)$ & $10(5.2)$ & 1.69 & $0.60-4.76$ & 0.312 \\
\hline \multicolumn{6}{|l|}{ Systemic disease, $n(\%)$} \\
\hline Diabetes mellitus & $28(14.5)$ & $55(28.4)$ & 2.33 & $1.40-3.88$ & $0.001 \ddagger$ \\
\hline Hypertension & $77(39.9)$ & $79(40.7)$ & 1.04 & $0.69-1.56$ & 0.869 \\
\hline Hypercholesterolemia & $9(4.7)$ & $11(5.7)$ & 1.23 & $0.49-3.04$ & 0.655 \\
\hline Ischemic heart disease & $14(7.3)$ & $19(9.8)$ & 1.39 & $0.68-2.86$ & 0.371 \\
\hline Cerebrovascular accident & $3(1.6)$ & $14(7.2)$ & 4.93 & $1.39-17.4$ & $0.007 \ddagger$ \\
\hline Migraine & $13(6.7)$ & $13(6.7)$ & 0.99 & $0.45-2.20$ & 0.989 \\
\hline Cold extremities & $15(7.8)$ & $22(11.3)$ & 1.52 & $0.76-3.02$ & 0.233 \\
\hline
\end{tabular}

Mean deviation refers to mean deviation in the Humphrey visual field analyzer C24-2. CCT, central corneal thickness; IOP, intraocular pressure; SE, spherical equivalent.

${ }^{*}$ Analysis by $\chi^{2}$ test except when analyzing age, mean deviation, mean, and interocular difference of CCT, IOP, and SE (Student's $t$-tests were used).

† IOP is the diurnal measurement before glaucoma medication for each patient.

$\ddagger P<0.05$.

mellitus with open-angle glaucoma was not consistently demonstrated in epidemiologic studies (some studies showed association, others did not), numerous clinical and experimental observations suggest a role for diabetes mellitus in NTG. The suggestion that diabetes mellitus is associated with impaired cerebrovascular reactivity and autoregulation in response to various stimuli has received general acceptance. ${ }^{22-24}$ Because impaired autoregulation is considered to be an important

TABLE 2. Comparisons between Eyes in the Unilateral and Bilateral NTG Groups

\begin{tabular}{|c|c|c|c|}
\hline \multirow[b]{2}{*}{ Candidate Variables } & \multicolumn{2}{|c|}{ Unilateral NTG Group } & \multirow[b]{2}{*}{$\boldsymbol{P}$} \\
\hline & Unaffected Eye & Affected Eye & \\
\hline IOP, mean (mm Hg) & $14.12 \pm 2.65$ & $14.49 \pm 2.57$ & 0.165 \\
\hline IOP fluctuation, mean (mm $\mathrm{Hg})$ & $2.39 \pm 1.51$ & $2.51 \pm 1.50$ & 0.529 \\
\hline CCT, mean $(\mu \mathrm{m})$ & $564.6 \pm 40.1$ & $559.1 \pm 39.8$ & 0.192 \\
\hline SE, mean (diopter) & $-0.51 \pm 1.05$ & $-0.50 \pm 1.02$ & 0.989 \\
\hline Mean deviation (dB) & - & $-3.2 \pm 3.5$ & \\
\hline \multirow[t]{2}{*}{ VCDR } & $0.58 \pm 0.16$ & $0.69 \pm 0.15$ & $<0.001$ \\
\hline & \multicolumn{2}{|c|}{ Bilateral NTG Group } & \\
\hline Candidate Variables & Less Affected Eye & First Affected Eye & $\boldsymbol{P}$ \\
\hline IOP, mean (mm Hg) & $14.85 \pm 2.86$ & $14.90 \pm 2.85$ & 0.840 \\
\hline IOP fluctuation, mean $(\mathrm{mm} \mathrm{Hg})$ & $2.44 \pm 1.11$ & $2.59 \pm 1.31$ & 0.375 \\
\hline $\mathrm{CCT}$, mean $(\mu \mathrm{m})$ & $563.9 \pm 38.0$ & $568.7 \pm 32.7$ & 0.204 \\
\hline SE, mean (diopter) & $-1.13 \pm 1.75$ & $-1.23 \pm 1.12$ & 0.898 \\
\hline Mean deviation (dB) & $-4.0 \pm 4.8$ & $-7.1 \pm 6.4$ & $<0.001$ \\
\hline VCDR & $0.68 \pm 0.18$ & $0.72 \pm 0.17$ & 0.039 \\
\hline
\end{tabular}

CCT, central corneal thickness; IOP, intraocular pressure; SE, spherical equivalent; VCDR, vertical cup-to-disc ratio. 
TABLE 3. Multivariate Risk Factors for Bilateral NTG

\begin{tabular}{|c|c|c|c|}
\hline Risk Factors & $\begin{array}{l}\text { Odds } \\
\text { Ratio }\end{array}$ & $\begin{array}{c}95 \% \\
\text { Confidence } \\
\text { Interval }\end{array}$ & $\boldsymbol{P}^{*}$ \\
\hline $\begin{array}{l}\text { Mean deviation (dB) by } 1-d B \\
\text { decrease }\end{array}$ & 1.19 & $1.13-1.25$ & $<0.001$ \\
\hline Mean IOP $>14 \mathrm{~mm} \mathrm{Hg}$ & 1.66 & $1.05-2.63$ & 0.030 \\
\hline Diabetes mellitus & 2.31 & $1.31-4.07$ & 0.004 \\
\hline Cerebrovascular accident & 4.27 & $1.07-16.9$ & 0.039 \\
\hline
\end{tabular}

Mean deviation refers to mean deviation in Humphrey visual field analyzer C24-2 in the first affected eye; mean IOP, mean IOP with diurnal measurements before glaucoma medication in the less affected eye.

* Age and sex are adjusted. The variables in the table are all in the same model and are independent predictors.

mechanism of eye damage in glaucoma, ${ }^{25,26}$ it is reasonable to suggest that diabetes mellitus may be associated with glaucoma. Further, experimental studies have reported that diabetes mellitus may induce retrograde axonal transport impairment $^{27}$ or may have an additive effect on retinal ganglion cell apoptosis induced by chronic elevation of IOP. ${ }^{28}$ The results of the present study yield an additional clinical observation supporting a role for diabetes mellitus in the pathophysiology of glaucoma.

In the present study, a history of cerebrovascular accident was also significantly associated with bilateral eye involvement in NTG. This is in line with recent reports indicating reduced cerebrovascular blood flow velocities in glaucoma patients. ${ }^{20,29}$ Moreover, it is considered that cerebrovascular disease is linked to vascular dysregulation, ${ }^{30,31}$ as is glaucoma. ${ }^{29,32,33}$ These observations together suggest that cerebrovascular disease and glaucoma may have a common pathologic mechanism that presumably involves reduced cerebral blood flow and impaired vascular dysregulation.

When both groups were analyzed together, the average IOP of the unaffected eyes in the unilateral NTG group was significantly lower than the IOP values of a randomly chosen eye from each patient in the bilateral NTG group. Moreover, mean IOP $>14 \mathrm{~mm} \mathrm{Hg}$ in the less affected eye was significantly associated with bilateral involvement in univariate and multivariate analyses, indicating that a relatively lower average IOP in the unaffected eye of the unilateral group may be associated with the absence of involvement of that eye in NTG, which in turn indicates that a certain level of IOP-related stress is needed for the development of optic neuropathy in NTG. This finding supports the current understanding that IOP is part of the pathogenic process in NTG. ${ }^{34}$

Mean IOP was not significantly higher, however, in the first affected eye compared with the contralateral less affected eye in bilateral NTG. This result is consistent with previous studies. Poinoosawmy et al. ${ }^{35}$ reported that a significant number of patients had higher IOP in the eye with less severe field loss. Orgül et al. ${ }^{36}$ reported no correlation between IOP and visual field damage or between interocular differences in IOP and visual field damage in NTG patients.

As described earlier, an IOP $>14 \mathrm{~mm} \mathrm{Hg}$ in the less affected eye was significantly associated with bilateral eye involvement. The cutoff of $14 \mathrm{~mm} \mathrm{Hg}$ of IOP was determined by repeated statistical trials. When the eye data were separated at an IOP of $14 \mathrm{~mm} \mathrm{Hg}$, the association between IOP and bilateral involvement became statistically significant. This observation carries the important implication that NTG develops less frequently in eyes with $\mathrm{IOP} \leq 14 \mathrm{~mm} \mathrm{Hg}$.

Although the difference in the IOP between unaffected eyes of the unilateral group and eyes from the bilateral group was statistically significant, it was only $0.8 \mathrm{~mm} \mathrm{Hg}$. Although we do not know whether this small difference is also clinically significant, observations from several large clinical trials suggest that it may be. For instance, the Early Manifest Glaucoma Trial reported that a $1-\mathrm{mm} \mathrm{Hg}$ increase in IOP on follow-up was associated with $10 \%$ increased risk for glaucoma progression. ${ }^{37}$ Moreover, in patients with advanced glaucoma, a 1-mm $\mathrm{Hg}$ increase in IOP fluctuation was associated with a $30 \%$ increased risk for glaucoma progression. ${ }^{38}$ Although these observations were made on patients with established glaucoma and were relevant to glaucoma progression, the result of those studies suggest that a $0.8-\mathrm{mm} \mathrm{Hg}$ difference in IOP may be significant in the development of glaucomatous damage. However, longitudinal studies involving larger numbers of samples may be needed to confirm the impact of IOP in the pathogenesis of NTG.

The magnitude of diurnal IOP fluctuation was not significantly higher in the affected eye than in the unaffected eye of the unilateral NTG group. In addition, there was no significant difference in diurnal IOP fluctuation between the unilateral and bilateral NTG groups. The role of diurnal IOP fluctuation in the pathogenesis of glaucoma is a matter of debate. Several studies have shown that large diurnal IOP fluctuation is an independent risk factor for progression of visual field damage. ${ }^{13,39}$ Recent studies have found, however, that the range of diurnal IOP variation was proportional to the level of IOP, indicating that diurnal variation in IOP was not in itself an independent risk factor for the progression of glaucoma. ${ }^{40}$ Our results support the negative association between diurnal IOP fluctuation and NTG.

The definition of "glaucoma at presentation" in the present study was based on characteristic glaucomatous optic nerve head damage and glaucomatous visual field loss. Because glaucomatous damage may exist in the presence of a normal field, ${ }^{41}$ some patients with "unilateral" glaucoma might have had optic disc damage in the contralateral eye that was classified as nonglaucomatous based on the normal visual field. Nevertheless, an important implication of the current data is that diabetes mellitus, cerebrovascular disease, and IOP greater than 14 $\mathrm{mm} \mathrm{Hg}$ in both eyes promotes bilateral disease progression such that both eyes reach the perimetric stage of disease earlier than occurs in patients without such factors.

The present study had several limitations. The analysis did not include all parameters indicated in the literature as NTG risk factors. Recently, fluctuation in mean ocular perfusion pressure, ${ }^{42}$ differences in immunologic markers such as antiphosphatidylserine antibodies, ${ }^{43}$ neuron-specific enolase autoantibodies, ${ }^{44}$ and variations in genetic polymorphisms ${ }^{45,46}$ have been suggested as risk factors for NTG. These systemic factors may be associated with bilateral eye involvement. Possible associations of these factors with bilateral eye involvement in NTG remain to be explored. Another limitation was that the study used predictive modeling and, thus, may be driven by the data. As such, the findings await confirmation in further studies. Last, the study involved only Korean patients. It appears there are significant variations in the prevalence of NTG between ethnic groups, ${ }^{47,48}$ suggesting groups may differ in terms of NTG pathogenesis or vulnerability. Thus, it is important that the present findings be verified in other populations before they can be considered to be broadly applicable.

It is acknowledged that IOP often, but not always, peaks at nighttime, when IOP measurements are not routinely obtained. Such an undetected IOP spike in one eye or both eyes may play a significant role in the development of glaucoma unilaterally or bilaterally. However, the possibility does not invalidate the findings of the present study. Although the nighttime IOP elevation may constitute an additional risk factor for NTG, inclusion of the nighttime IOP profile in the regression analysis 
would not negate the factors identified in the present study as long as they are not dependent on the nighttime IOP profile, which is likely to be the case.

It may be argued that definitive diagnoses of NTG could not be made in our patients because we did not measure IOP at night. However, it is the general practice to make the diagnosis of NTG based on the daytime IOP. ${ }^{34,49,50}$ As such, the diagnosis of NTG in our patients may be acceptable. Moreover, we believe the definition of NTG does not provide a source of bias in the present study because we diagnosed NTG based on the same criteria in the unilateral and bilateral groups.

Onset of visual field loss occurred more often in the left eye, and greater field defect was more likely to develop in this eye than the right eye. This is in line with previous findings. ${ }^{35,51}$ Among patients with greater field defects in left eyes, $47 \%$ in the unilateral NTG group and 33\% in the bilateral NTG group had higher IOP in the left eyes. Thus, the left eye predilection could not be explained by intereye asymmetry of IOP, as also noted in previous studies. ${ }^{35,51}$ At present, it is not clear why left eyes of NTG patients often develop visual field defects earlier than do right eyes.

In conclusion, our study demonstrates that disease severity of the first affected eye, diabetes mellitus, previous cerebrovascular accident, and IOP higher than $14 \mathrm{~mm} \mathrm{Hg}$ in both eyes are associated with bilateral eye involvement in NTG. These data suggest that systemic vascular factors and IOP play significant roles in the pathogenesis of NTG. It appears that although systemic vascular factors increase the susceptibility of the optic nerve, a certain level of pressure-induced stress is also required for glaucomatous optic neuropathy.

\section{References}

1. Klein B, Klein R, Sponsel WE, et al. Prevalence of glaucoma: the Beaver Dam Eye Study. Ophthalmology. 1992;99:1499-1504.

2. Leske MC, Connell AM, Wu SY, et al. Incidence of open-angle glaucoma: the Barbados Eye Studies. Arch Ophthalmol. 2001;119: 89-95.

3. Tielsch JM, Sommer A, Katz J, et al. Racial variations in the prevalence of primary open-angle glaucoma: the Baltimore Eye Survey. JAMA. 1991;266:369-374.

4. Tielsch JM, Katz J, Sommer A, et al. Family history and risk of primary open-angle glaucoma: the Baltimore Eye Survey. Arch Ophthalmol. 1994;112:69-73.

5. Perkins ES, Phelps CD. Open angle glaucoma, ocular hypertension, low tension glaucoma and refraction. Arch Ophthalmol. 1982;100: $1464-1467$.

6. Chihara E, Liu X, Dong J, et al. Severe myopia as a risk factor for progressive visual field loss in primary open-angle glaucoma. Ophthalmologica. 1997;211:66-71.

7. Mitchell P, Smith W, Chey T, Healey PR. Open-angle glaucoma and diabetes: the Blue Mountains Eye Study, Australia. Ophthalmology. 1997;104:712-718.

8. Dielemans I, de Jong PT, Stolk R, et al. Primary open-angle glaucoma, intraocular pressure, and diabetes mellitus in the general elderly population: the Rotterdam Study. Ophthalmology. 1996; 103:1271-1275.

9. Bonovas S, Peponis V, Filioussi K. Diabetes mellitus as a risk factor for primary open-angle glaucoma: a meta-analysis. Diabet Med. 2004;21:609-614.

10. Harrington DO. The pathogenesis of the glaucoma field. Am J Ophthalmol. 1959;47:177-185.

11. Ishida K, Yamamoto T, Sugiyama K, Kitazawa Y. Disk hemorrhage is a significantly negative prognostic factor in normal-tension glaucoma. Am J Ophthalmol. 2000;129:707-714.

12. Jonas JB, Xu L. Optic disc hemorrhages in glaucoma. Am J Ophthalmol. 1994;118:1-8.

13. Asrani S, Zeimer R, Wilensky J, et al. Large diurnal fluctuations in intraocular pressure are an independent risk factor in patients with glaucoma. J Glaucoma. 2000;9:134-142.
14. Gordon MO, Beiser JA, Brandt JD, et al. The Ocular Hypertension Treatment Study: baseline factors that predict the onset of primary open-angle glaucoma. Arch Ophthalmol. 2002;120:714-720.

15. Phelps CD, Corbett JJ. Migraine and low-tension glaucoma: a casecontrol study. Invest Ophthalmol Vis Sci. 1985;26:1105-1108.

16. Broadway DC, Drance SM. Glaucoma and vasospasm. Br J Ophthalmol. 1998;82:862-870.

17. Hayreh SS, Zimmerman MB, Podhajsky P, Alward WL. Nocturnal arterial hypotension and its role in optic nerve head and ocular ischemic disorders. Am J Ophthalmol. 1994;117:603-624.

18. Marcus DM, Costarides AP, Gokhale P, et al. Sleep disorders: a risk factor for normal-tension glaucoma? J Glaucoma. 2001;10:177183.

19. Girkin CA, McGwin G Jr, McNeal SF, Owsley C. Is there an association between pre-existing sleep apnoea and the development of glaucoma? Br J Ophthalmol. 2006;90:679-681.

20. Sugiyama $\mathrm{T}$, Utsunomiya $\mathrm{K}$, Ota $\mathrm{H}$, et al. Comparative study of cerebral blood flow in patients with normal-tension glaucoma and control subjects. Am J Ophthalmol. 2006;141:394-396.

21. Caprioli J. Automated perimetry on glaucoma. Am J Ophthalmol. 1991;111:235-239.

22. Dandona P, James IM, Newbury PA, et al. Cerebral blood flow in diabetes mellitus: evidence of abnormal cerebrovascular reactivity. BMJ. 1978;2:325-326.

23. Bentsen N, Larsen B, Lassen NA. Chronically impaired autoregulation of cerebral blood flow in long-term diabetics. Stroke. 1975;6: 497-502.

24. Pallas F, Larson DF. Cerebral blood flow in the diabetic patient. Perfusion. 1996;11:363-370.

25. Grieshaber MC, Flammer J. Blood flow in glaucoma. Curr Opin Ophthalmol. 2005;16:79-83.

26. Flammer J, Orgul S, Costa VP, et al. The impact of ocular blood flow in glaucoma. Prog Retin Eye Res. 2002;21:359-393.

27. Zhang L, Ino-ue M, Dong $\mathrm{K}$, Yamamoto M. Retrograde axonal transport impairment of large- and medium-sized retinal ganglion cells in diabetic rat. Curr Eye Res. 2000;20:131-136.

28. Kanamori A, Nakamura M, Mukuno $\mathrm{H}$, et al. Diabetes has an additive effect on neural apoptosis in rat retina with chronically elevated intraocular pressure. Curr Eye Res. 2004;28:47-54.

29. Harris A, Zarfati D, Zalish M, et al. Reduced cerebrovascular blood flow velocities and vasoreactivity in open-angle glaucoma. Am J Ophthalmol. 2003;135:144-147.

30. Fu JH, Lu CZ, Hong Z, et al. Relationship between cerebral vasomotor reactivity and white matter lesions in elderly subjects without large artery occlusive disease. J Neuroimaging. 2006;16:120125.

31. Ainslie PN, Murrell C, Peebles K, et al. Early morning impairment in cerebral autoregulation and cerebrovascular $\mathrm{CO}_{2}$ reactivity in healthy humans: relation to endothelial function. Exp Physiol. 2007;92:769-777.

32. Drance SM, Douglas GR, Wijsman K, et al. Response of blood flow to warm and cold in normal and low-tension glaucoma patients. Am J Ophthalmol. 1988;105:35-39.

33. Hosking SL, Harris A, Chung HS, et al. Ocular haemodynamic responses to induce hypercapnia and hyperoxia in glaucoma. $\mathrm{BrJ}$ Ophthalmol. 2004;88:406-411.

34. Collaborative Normal-Tension Glaucoma Study Group. Comparison of glaucomatous progression between untreated patients with normal-tension glaucoma and patients with therapeutically reduced intraocular pressures. Am J Ophthalmol. 1998;126:487497.

35. Poinoosawmy D, Fontana L, Wu JX, et al. Frequency of asymmetric visual field defects in normal-tension and high-tension glaucoma. Ophthalmology. 1998;105:988-991.

36. Orgul S, Flammer J. Interocular visual-field and intraocular-pressure asymmetries in normal-tension-glaucoma. Eur J Ophthalmol. 1994;4:199-201.

37. Leske MC, Heijl A, Hussein M, et al. Factors for glaucoma progression and the effect of treatment: the early manifest glaucoma trial. Arch Ophthalmol. 2003;121:48-56. 
38. Nouri-Mahdavi K, Hoffman D, Coleman AL, et al. Predictive factors for glaucomatous visual field progression in the Advanced Glaucoma Intervention Study. Ophthalmology. 2004;111:1627-1635.

39. Ishida K, Yamamoto T, Kitazawa Y. Clinical factors associated with progression of normal-tension glaucoma. J Glaucoma. 1998;7: 372-377.

40. Brengtsson B, Heiji A. Diurnal IOP fluctuation: not an independent risk factor for glaucomatous visual field loss in high-risk ocular hypertension. Graefes Arch Clin Exp Opbthalmol. 2005;243:513518.

41. Quigley HA, Addicks EM, Green WR. Optic nerve damage in human glaucoma, III: quantitative correlation of nerve fiber loss and visual field defect in glaucoma, ischemic neuropathy, papilledema, and toxic neuropathy. Arch Opbthalmol. 1982;100:135146.

42. Sehi M, Flanagan JG, Zeng L, et al. Relative change in diurnal mean ocular perfusion pressure: a risk factor for the diagnosis of primary open-angle glaucoma. Invest Opbthalmol Vis Sci. 2005;46:561567.

43. Kremmer S, Kreuzfelder E, Klein R, et al. Antiphosphatidylserine antibodies are elevated in normal tension glaucoma. Clin Exp Immunol. 2001;125:211-215.

44. Maruyama I, Ikeda Y, Nakazawa M, Ohguro H. Clinical roles of serum autoantibody against neuron-specific enolase in glaucoma patients. Toboku J Exp Med. 2002;197:125-132.
45. Kim SH, Kim JY, Kim DM, et al. Investigations on the association between normal tension glaucoma and single nucleotide polymorphisms of the endothelin-1 and endothelin receptor genes. $\mathrm{Mol}$ Vis. 2006;12:1016-1021.

46. Mabuchi F, Tang S, Kashiwagi K, et al. The OPA1 gene polymorphism is associated with normal tension and high tension glaucoma. Am J Ophthalmol. 2007;143:125-130.

47. Shiose Y, Kitazawa Y, Tsukahara S, et al. Epidemiology of glaucoma in Japan: a nationwide glaucoma survey. Jpn J Opbthalmol. 1991;35:133-155.

48. Sommer A, Tielsh JM, Katz J, et al. Relationship between intraocular pressure and primary open angle glaucoma among white and black Americans: the Baltimore Eye Survey. Arch Opbthalmol. 1991;109:1090-1095.

49. Su WW, Cheng ST, Hsu TS, Ho WJ. Abnormal flow-mediated vasodilation in normal-tension glaucoma using a noninvasive determination for peripheral endothelial dysfunction. Invest $O p b$ thalmol Vis Sci. 2006;47:3390-3394.

50. Ngan R, Lam DL, Mudumbai RC, Chen PP. Risk factors for noncompliance with follow-up among normal-tension glaucoma suspects. Am J Ophthalmol. 2007;144:310-311.

51. Crichton A, Drance SM, Douglas GR, Schulzer M. Unequal intraocular pressure and its relation to asymmetric visual field defects in low-tension glaucoma. Ophthalmology. 1989;96:1312-1314. 